
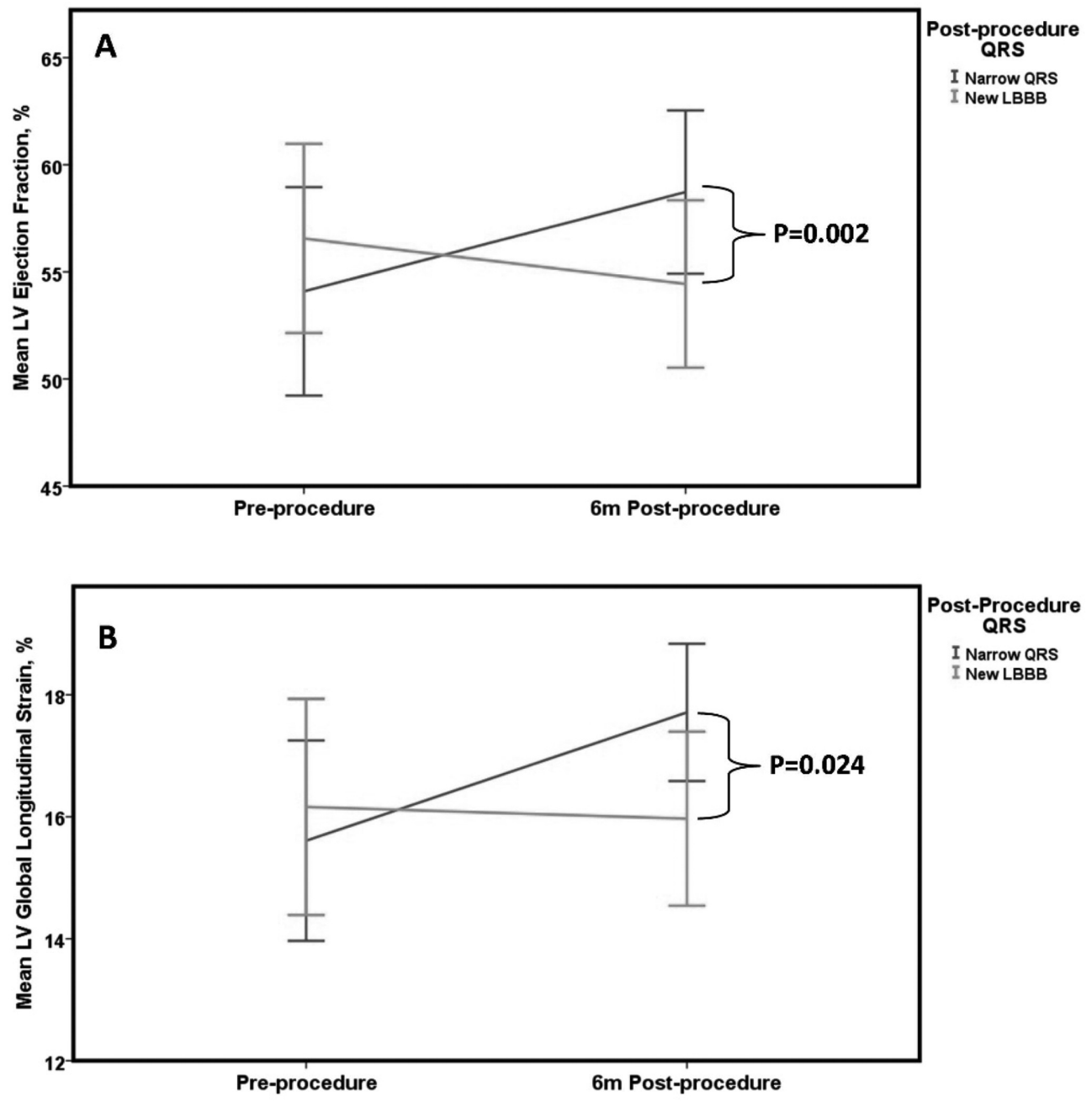

Abstract 39 Figure 1 Change in LVEF \& GLS according to post-TAVI QRS

$\left.10.2 \mathrm{ml} / \mathrm{m}^{2}, \mathrm{p}=0.02\right)$. Further CMR characteristics can be seen in Table 1 . Those with LBBB-T exhibited significant interventricular dyssynchrony $6 \mathrm{~m}$ follow up compared with the nQRS population (LBBB-T $130 \pm 73 \mathrm{~ms}$ vs nQRS $23 \pm 86$ $\mathrm{ms}, \mathrm{p}=<0.001)$. Intraventricular dyssynchrony was also demonstrated in the LBBB-T at $6 \mathrm{~m} ; 118 \pm 103 \mathrm{~ms}$ compared with $13 \pm 106 \mathrm{~ms}(\mathrm{p}=0.001)$ in the nQRS group. There was a significant correlation between post procedure QRS and interventricular and intraventricular dyssynchrony $(\mathrm{r}=0.57$, $\mathrm{p}=<0.001$ and $\mathrm{r}=0.49, \mathrm{p}=<0.001$ respectively). Neither group experienced any change in right ventricular longitudinal function (nQRS $21.7 \pm 7.0$ to $21.5 \pm 6.2 \mathrm{~mm}, \mathrm{p}=0.817$ LBBB-T $18.9 \pm 5.8$ to

$18.6 \pm 5.8 \mathrm{~mm}, \mathrm{p}=0.773)$. Post-procedure aortic regurgitant fraction was similar between groups (nQRS $5.4 \pm 5.7$ vs LBBB-T $5.5 \pm 3.3 \%, \mathrm{p}=0.948$ ). There was an inverse correlation between QRS duration and change in LVEF $(r=-0.46, p$ $=0.001)$ and QRS duration and change in LV GLS $(r=-0.37$, $\mathrm{p}=0.010)$.

Conclusion LBBB-T is associated with less favourable cardiac reverse remodelling at medium term follow up. In view of this, every effort should be made to prevent TAVI-induced LBBB, especially as TAVI is extended to a younger, lower risk population.

\section{QUANTIFICATION OF AORTIC REGURGITATION FOLLOWING TRANSCATHETER AORTIC VALVE IMPLANTATION (TAVI): A CMR STUDY OF TWO PROSTHESIS DESIGNS}

${ }^{1}$ Tarique Al Musa*, ${ }^{1}$ Akhlaque Uddin, 'Laura E Dobson, ${ }^{1}$ Peter P Swoboda, ${ }^{1}$ Pankaj Garg, ${ }^{1}$ Graham J Fent, ${ }^{1}$ James RJ Foley, ${ }^{2}$ Christopher J Malkin, ${ }^{1}$ Sven Plein, ${ }^{2}$ Daniel J Blackman, ${ }^{1}$ John P Greenwood. 'University of Leeds; ' Leeds Teaching Hospitals; *Presenting Author

\subsection{6/heartjnl-2016-309890.40}

Background Transcatheter aortic valve implantation is an established therapeutic option for patients with severe symptomatic aortic stenosis unsuitable for surgery. CMR has a lower intraobserver and inter-observer variability than echocardiography in the assessment of aortic regurgitation (AR), offering full volumetric quantitation independent of the number or eccentricity of regurgitant jets. Moderate AR following TAVI is an independent predictor of mortality in the postoperative period and has prompted development of novel bioprostheses designed to facilitate precise deployment and minimise paravalvular regurgitation (Figure 1).

Aim To compare the degree of early post-procedure aortic regurgitation following implantation of the Medtronic CoreValve and Boston Scientific Lotus Valve systems in patients treated for severe symptomatic aortic stenosis.

Methods All patients underwent an identical 1.5T CMR scan (Intera, Phillips Healthcare, Best, Netherlands) post-procedure 
prior to discharge. Through-plane phase contrast velocity encoded imaging was performed perpendicular to the aortic valve jet at a level above the aortic prosthesis. Aortic flow was subsequently quantified offline (QFlow version 7.2, Medis Medical Imaging Systems, Leiden, The Netherlands) and regurgitant fraction (\%) derived. AR was defined as regurgitant fraction of none/trivial $\leq 5 \%$, mild $6-15 \%$, moderate $16-$ $25 \%$, moderate-severe $26-48 \%$, and severe $>48 \%$ The invasive $\mathrm{AR}$ index derived at the time of TAVI was also recorded ([(Diastolic BP-LVEDP)/Systolic BP] x100).

Results 18 Medtronic CoreValve patients (age $79.6 \pm 6.3$ years, 67\% male, EuroSCORE II $4.59 \pm 3.59 \%$ ) and 24 Boston Lotus TAVI patients (age $78.6 \pm 8.7$ years, 54\% male, EuroSCORE II $4.07 \pm 3.37 \%$ ) were studied at a median interval of 6 days following implantation. The residual peak aortic pressure gradient observed following Boston Lotus implantation was higher than that following Medtronic CoreValve $(25.8 \pm 12.1$ vs. $15.0 \pm 5.5 \mathrm{mmHg}, \mathrm{p}=0.001)$. The absolute regurgitant fraction was significantly lower following Boston Lotus implantation than following Medtronic CoreValve (Lotus: $4.0 \pm 3.5 \%$ vs. CoreValve: $11.7 \pm 7.2 \%, \mathrm{p}=$ 0.001). The classification of AR severity is summarised in Figure 2. Overall, 39\% of patients with CoreValve had moderate AR compared to no reported cases in the Lotus group. There was a negative correlation between invasive AR index and regurgitant fraction from CMR imaging (Spearman's coefficient $-0.4, \mathrm{p}=0.016$ ).

Conclusions CMR directly quantifies AR following TAVI and there is agreement with invasive AR measurements. There was

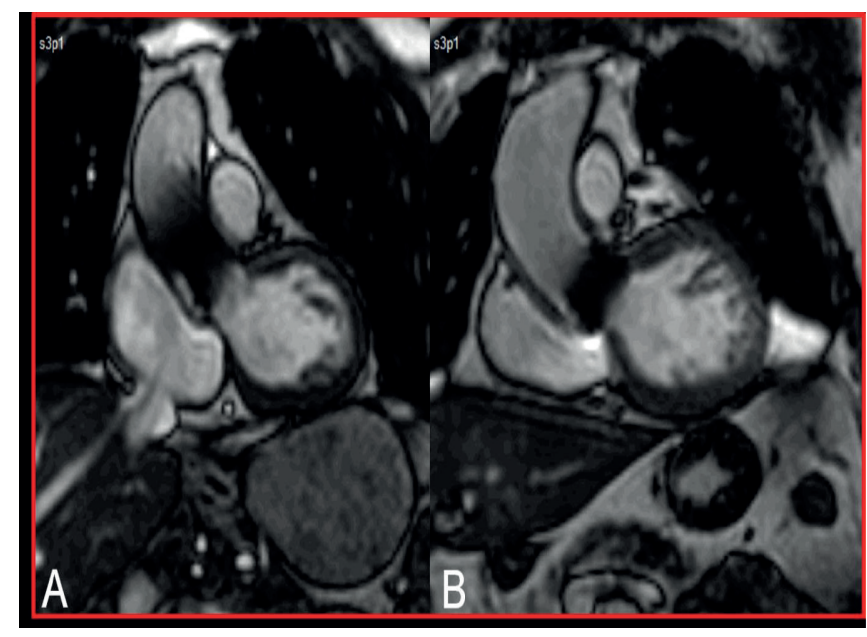

Abstract 40 Figure 1 Coronal CMR images following implantation of the Medtronic CoreValve (A) and the Boston Lotus (B) TAVI prostheses

significantly less aortic regurgitation in the immediate postimplant period using the Boston Lotus valve compared with the Medtronic CoreValve. However the residual peak pressure gradient was lower with the Medtronic CoreValve. Further work is required to assess the longer term differences in AR and whether this translates into a mortality benefit.

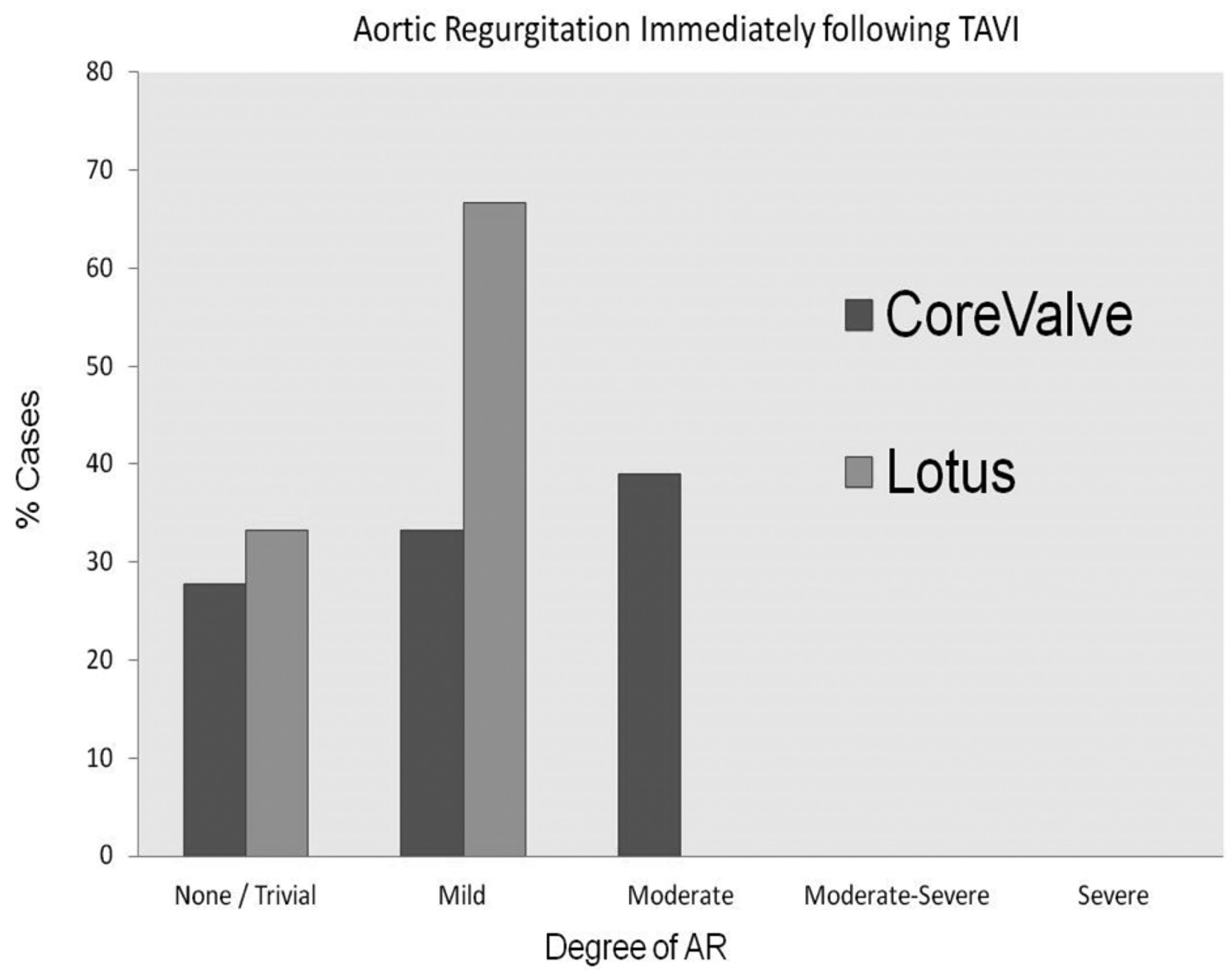

Abstract 40 Figure 2 Classification of AR severity seen following implantation of two different TAVI valve types 